\title{
LA ORIENTACIÓN PROFESIONAL EN LA UNIVERSIDAD, UN FACTOR DE CALIDAD SEGÚN LOS ALUMNOS
}

\author{
VOCATIONAL CONSELING AT THE UNIVERSITY, A QUALITY FACTOR \\ FROM THE STUDENTS POINT OF VIEW
}

\author{
Ignacio González López* y Juan Francisco Martín Izard** \\ Universidad de Córdoba y Universidad de Salamanca
}

\begin{abstract}
RESUMEN
En este artículo se trata la orientación profesional en la universidad como un factor de calidad. En la investigación que aquí se refleja recogemos la demanda de los alumnos de la Universidad de Salamanca que consideran la orientación profesional como un elemento que favorece su incursión en el mercado laboral de forma exitosa y, por lo tanto, como un indicador de la calidad de la universidad. Tras la exposición de alguna de las ideas que nos parecen relevantes al respecto de comprender que la orientación profesional en este sentido, hemos llevado a cabo un estudio en el que se refleja la opinión de los estudiantes sobre este tema. Para ello, nos hemos centrado en las cualidades y requisitos necesarios para acceder a un empleo, el conocimiento de las salidas profesionales de las diferentes titulaciones y la valoración de los sistemas de orientación profesional existentes en la universidad.
\end{abstract}

Palabras clave: Orientación profesional, Universidad de calidad, estudiante universitario, indicadores.

\begin{abstract}
In this article it is the professional orientation in the university like a factor of quality. In the investigation that here is reflected we pick up the demand of the students of the University of Salamanca that they consider the professional orientation as an element that favors their incursion in the labor market in a successful way and, therefore, as an indicator of the quality of the university. After the exhibition of some of the ideas that we find outstanding in this respect of understanding that the professional orientation in this sense, we have carried out a study in which is reflected the opinion of the students on this topic. For it, we have centered ourselves in the qualities and necessary requirements to consent to an employment, the knowledge of the professional exits of the different students and the valuation of the existent systems of professional orientation in the university.
\end{abstract}

Key words: Professional orientation, University of quality, university student, indicators

\footnotetext{
* Universidad de Córdoba.

** Profesor del Departamento de Didáctica, Organización y Métodos de Investigación de la Universidad de Salamanca.
} 


\section{Introducción}

Desde la perspectiva de los profesionales de la orientación, tanto desde la práctica como desde la investigación, se considera que la Universidad, como institución formadora de profesionales cualificados, debe dedicar parte de sus esfuerzos a la orientación profesional de los alumnos. Sin embargo, esta opinión generalizada de los expertos no parece verse reflejada en las actuales políticas universitarias, así como la orientación profesional en la universidad no es considerada como un factor de calidad de la institución. En este artículo se verá reflejada la opinión de los principales usuarios de la oferta universitaria, los alumnos, que coinciden con la opinión de los expertos.

La orientación en la universidad es considerada como un factor imprescindible para la mejora de la calidad de la enseñanza por numerosos autores (Díaz Allué, 1989; Apodaca y Lobato, 1997; García Basallote et al., 1999; López Franco y Oliveros, 1999 y Rodríguez Espinar, 1989) y puesto de manifiesto en el documento que sirvió como elemento desencadenante del debate acerca de la Universidad, el Informe Universidad 2000 (Bricall, 2000). En su apartado tercero, en el que se exponen las necesidades y tendencias de la difusión del conocimiento y cómo formar para aprender, aparece un apartado denominado "asesoramiento", donde se expone la importancia de la orientación y la tutoría en la universidad.

La actual complejidad del mundo laboral y la consecuente complejidad de las titulaciones universitarias, con nuevas ofertas, variados itinerarios formativos, gran optatividad, etc., hacen que para el alumno sea más difícil tomar decisiones y buscar aquellas ofertas formativas más adecuadas a sus necesidades, para lo que debe existir un sistema de orientación profesional dentro de la institución que le ayude, asesore y guíe en este proceso.

Esta situación es percibida por los alumnos, que consideran a la orientación profesional como indicador de una institución universitaria de calidad. Según mostraremos, los estudiantes demandan dicha orientación en la Universidad, así como formación en aspectos complementarios que les facilite el acceso a un empleo.

Esta demanda por parte de los alumnos responde a una organización educativa inmersa en una sociedad cambiante que debe plantearse nuevos objetivos en función de las nuevas necesidades. En primer lugar, expondremos algunas ideas que nos parecen relevantes al respecto para llegar a comprender que la orientación profesional debe ser considerada como un factor de calidad en la Universidad para, posteriormente, presentar los resultados de una investigación en la que se refleja la opinión de los estudiantes en este sentido.

\section{Los objetivos de la educación superior}

La Universidad española está en pleno proceso de cambio, se acaba de aprobar una nueva Ley de Universidades (Ley Orgánica de Universidades, 2001) y se está desarrollando un nuevo marco legislativo que, aun introduciendo novedades importantes, sigue manteniendo como funciones de esta institución la investigación, la innovación, la enseñanza y la formación, la educación permanente y la cooperación internacional. 
Esta situación, entre otras razones, responde a un cambio social caracterizado, tal y como ya destacó la OCDE (1991), por la revolución tecnológica, el desarrollo económico y la masificación universitaria.

Desde esta perspectiva, la Universidad debe formar a sus estudiantes para que puedan atender a las demandas interpuestas por la evolución del mercado laboral. De manera continua aumentan las exigencias de cualificación, así como la evolución de las tecnologías aplicadas a las diferentes profesiones, del mismo modo que se busca personal con iniciativa, capaz de tomar decisiones y de resolver problemas. No debemos olvidar que la Universidad tiene entre sus funciones la formación de profesionales altamente cualificados y que, entre otros aspectos, deben estar preparados para afrontar estas demandas del mercado de trabajo. Estos contenidos son propios de la orientación profesional.

Los cambios citados hasta este momento plantean a los responsables de las nuevas políticas universitarias mecanismos de respuesta. Según Abrile (1994: 13), los objetivos para este nivel educativo han de ser:

- Preparar ciudadanos capaces de convivir en sociedades marcadas por la diversidad, capacitándolos para incorporar las diferencias, de manera que contribuyan a la integración a y a la solidaridad, así como para enfrentar la fragmentación y la segmentación que amenaza a muchas sociedades en la actualidad.

- Formar recursos humanos que respondan a los nuevos requerimientos del proceso productivo y a las formas de organización del trabajo resultantes de la revolución tecnológica.

- Capacitar al conjunto de la sociedad para convivir con la racionalidad de las nuevas tecnologías, transformándolas en instrumentos que mejoren la calidad de vida.

En este sentido, no podemos obviar los enormes beneficios que tanto para los ciudadanos en particular como para la sociedad en general debe tener la educación superior. Admitida esta prerrogativa desde los estudios de Astin y Henson (1977) o Haveman y Wolfe (1984), Mora Ruiz (1991: 28-31) evidenció su existencia en dos aspectos:

a) Beneficios individuales, concretados en beneficios educativos (capacidades, conocimientos, valores, actitudes, intereses, hábitos, etc.), beneficios marginales (ventajas sociales y económicas que, en principio, no tiene que ver con su formación específica), y beneficios existenciales (producidos por la experiencia universitaria vivenciada).

b) Beneficios sociales, reflejados en un apoyo al progreso social propiciando la igualdad social, la investigación sobre temas sociales de gran relevancia, contribuyendo al crecimiento económico y fomentando el desarrollo cultural.

Para que estos beneficios personales y sociales puedan materializarse es necesario plantearnos que la Universidad atienda a la construcción de los proyectos profesionales de los alumnos, ayudándoles en la búsqueda de itinerarios formativos, en la toma de decisiones, en el conocimiento de la realidad laboral, capacitándoles para localizar y manejar información, para desarrollar proyectos, para el trabajo colaborativo, etc.

Estas demandas han de ser atendidas en un proceso continuo y programado (López Franco y Oliveros, 1999), por lo que estos contenidos deben tratarse tanto desde las propias asignaturas como específicamente con un currículo propio de orientación profesional. (Rodríguez Diéguez, 2002 y Romero y Sobrado, 2002) 


\section{Nuevos alumnos y nuevos objetivos de la orientación profesional en la Universidad de calidad}

El principal afectado por las políticas de calidad en la Universidad es el alumno como beneficiario de sus planes de formación. Por esta razón, hemos de aclarar que el concepto tradicional de alumno universitario ha cambiado. Como apuntaba Moncada (1971), "con frecuencia los autores, al examinar las cuestiones del alumnado, tienen en mente un tipo de estudiante o, mejor dicho, un tipo de presencia estudiantil en la Universidad que ya no representa ni siquiera a la mayoría de las personas que siguen estudios superiores en los países occidentales" (Moncada, 1971: 175). Más allá del convencional alumno joven, podemos encontrarnos con aquellos que regresan a las aulas universitarias en busca de un horizonte intelectual más amplio o para mejorar su propio status y sus expectativas en el mercado de empleo.

Estos alumnos se convierten, de algún modo, en universitarios con dedicación exclusiva. Junto a ellos tenemos a los que hacen compatibles sus estudios con otra ocupación, principalmente laboral, que les lleva a modificar el ritmo normal de dedicación a los estudios para acomodarlos a esta situación.

Además, un gran número de personas pueden considerarse como alumnos universitarios en tanto tratan de conseguir un incremento de su cultura o de sus habilidades, asistiendo a programas de perfeccionamiento desde la perspectiva de la educación superior, pero alejados de la obtención de un título académico.

Por otro lado, también son alumnos universitarios aquellos que han decidido minimizar el contacto con la institución y las relaciones personales que en ella se establecen desde los ámbitos de la universidad abierta, la educación a distancia y la enseñanza virtual.

En cualquier caso, el universitario debe ser una persona que, al concluir esta etapa de su vida, posea un conocimiento científico de su actividad, es decir, aquella formación básica que le permita desarrollar las demandas de la profesión a ejercer. Al mismo tiempo, se espera de él un comportamiento acorde con un estándar asociado, no tanto con la formación académica recibida, como con las vivencias culturales, emocionales y sociales experimentadas en la Universidad.

Esta concepción de lo que es el alumno universitario nos lleva a plantearnos los objetivos que ha de cumplir la institución universitaria. Señalamos algunas referencias en este sentido.

Hernández Pina (1997: 30), citando los trabajos de Atkins, Beattie y Dockrell (1993), señala cuatro objetivos complementarios que pueden dar lugar a las potencialidades de la formación de los alumnos, del contenido de un curso o de una institución:

1. Participar de una experiencia educativa general, adquiriendo conocimientos y desarrollándose cognitiva y personalmente como adulto.

2. Preparación para el conocimiento creativo, su aplicación y su difusión, adquiriendo un marco conceptual, unos procedimientos, metodologías, técnicas, etc.

3. Preparación para una profesión específica u ocupación profesional, integrando teoría y práctica, adquiriendo experiencias, desarrollando habilidades, competencias, actitudes y comprendiendo el contexto propio de la profesión. 
4. Preparación para el empleo en general, desarrollando habilidades diversas y trabajando en contextos variados.

Del mismo modo, Chadwick y Thorne de Trelles (1998) han tratado de buscar una forma de especificar detalladamente estos contenidos a través de una ponderación de variables. Destacan aquellas que están relacionadas con la capacidad para resolver problemas, la transferencia de los aprendizajes a situaciones de vida real y la construcción de un espíritu crítico.

Álvarez Rojo et al. (1999) llegan a estructurar una serie de categorías básicas que exige el trabajo de los alumnos universitarios, tales como el autoconcepto (conocimiento de sí mismo y autoestima), las actitudes frente a los estudios (vocación, motivación e interés por el trabajo), dedicación al estudio (asistencia a clase y constancia), el estilo de trabajo (responsabilidad, autonomía y adaptabilidad), las habilidades para la asimilación de contenidos (habilidad natural de los sujetos, habilidad para captar la información ofrecida por el profesor, habilidad para identificar los aspectos fundamentales, habilidad para sintetizar, habilidad para esquematizar, habilidad para ampliar información, habilidad para retener la información, habilidad para la resolución de dudas y habilidad para buscar experiencias prácticas complementarias), las habilidades de organización (identificación de las exigencias de cada materia y planificación del trabajo) y las habilidades sociales (capacidad de relación con los compañeros, capacidad para relacionarse con el profesorado y competitividad).

Centrándonos en los objetivos que debe tener la orientación en la universidad, Rodríguez Diéguez (2002) señala los siguientes:

1. Autoconocimiento y relaciones interpersonales, fomentando competencias que inciden en el desarrollo integral de la persona desde una perspectiva individual y social.

2. Exploración y planificación de la carrera, desarrollando la capacidad de conocimiento del entorno sociolaboral, el análisis del mismo y la toma de decisiones.

3. Desarrollo educativo y ocupacional, concibiendo la educación como el resultado de la interacción entre el currículum instructivo y el orientador.

Por último, Romero y Sobrado (2002) proponen una introducción de la orientación en el currículo académico universitario con los siguientes objetivos:

1. Facilitar el desarrollo de la identidad personal y profesional, favoreciendo la seguridad afectiva, la autoestima y adquiriendo una posición propia, coherente y consecuente con la profesión.

2. Aprender a dar sentido a la propia experiencia y a construir proyectos profesionales y vitales, desarrollando la autonomía, la conciencia crítica y autorreflexiva y aprendiendo a vivir en la ambigüedad y movilidad.

3. Facilitar el desarrollo de una acción colectiva social y política transformadora de construcción y creación de las condiciones que permitan el desarrollo.

\section{La orientación en la universidad como factor de calidad}

La orientación profesional en la universidad es, por lo tanto, un objetivo fundamental que debe cumplir esta institución, sin embargo, en el Plan Nacional de Calidad de las Universidades (1995) se puede apreciar que no se contemplan estos aspectos como indicadores 
de calidad, quedando la evaluación reducida a unos cuantos datos cuantitativos que, aunque importantes, no aportan toda la información necesaria para definir, evaluar y mejorar la calidad de la formación recibida por los estudiantes universitarios $\mathrm{y}$, en última instancia, conseguir una Universidad de calidad. No dejan de ser indicadores, en su mayoría de rendimiento, entendiendo estos como medidas objetivas, usualmente cuantitativas, de los logros de una institución o de todo un sistema de educación superior (Ball y Halwachi, 1987), que dan lugar a reivindicaciones por parte de la población estudiantil en cuanto a la clarificación de su perfil profesional, la gestión del personal y los recursos disponibles, la metodología docente y las calificaciones alcanzadas en las distintas materias, entre otras.

No se da pie a valorar, según las indicaciones de Gento Palacios (1996) procedimientos (creatividad, orden, disciplina, autoorganización, técnicas de estudio, trabajo en equipo, manejo de materiales, recursos de información y nuevas tecnologías, resolución de problemas...), actitudes (intereses, curiosidades, respeto, tolerancia, espíritu crítico, rigor intelectual, aprendizaje permanente...), habilidades sociales y morales (civismo, solidaridad, amistad, respeto a la igualdad, tolerancia, inserción cultural, cumplimiento de obligaciones, colaboración, autonomía, libertad, honestidad, esfuerzo, sinceridad...), etc., los cuales, junto a los indicadores de rendimiento, han de satisfacer unas necesidades que, en términos de Maslow (1991) y ahondando en la perspectiva de la Calidad Total ya mencionada, comienzan con la subsistencia y concluyen con la autorrealización, donde el alumno, libre y autónomo, es consciente de sus responsabilidades y capaz de tomar sus propias decisiones. Se trata de elementos inmersos dentro de la formación de todo estudiante universitario, pero que no son objeto de evaluación sistematizada por parte de las administraciones competentes.

Existen actualmente diferentes modelos y enfoques en torno a las dimensiones que definen lo que ha de constituirse como una Universidad de calidad (modelo del premio Deming, modelo del premio Baldrige, modelo de las normas ISO y modelo europeo de gestión de calidad, entre otros), destacando de ellos una serie de elementos que, con especial referencia al alumno, condicionan esta definición:

1. Las características personales de los sujetos.

2. Los elementos que definen el medio educativo en el que se desarrolla la actividad académica de los estudiantes. nivel educativo y tipo de titulación.

3. Las condiciones en las que se produce el acceso a la Universidad: vía de ingreso en la Universidad, rendimiento previo y motivos de elección de estudios.

4. Los objetivos de la institución universitaria, que definen su carácter y ayudan a aportar criterios para su definición Un elemento a valorar en las políticas de calidad es el cumplimiento de estos fines (Mora Ruiz, 1991), para lo cual, el alumno ha de ser un buen contribuyente.

5. La asimilación por parte de los estudiantes del rol que supone ser un estudiante universitario, elemento que condiciona la perspectiva que los diferentes alumnos tengan de la institución en la que estudian.

6. La participación del alumnado, considerada como un medio de formación y no sólo como mecanismo de gestión (San Fabián Maroto, 1997: 15). Debe impregnar el conjunto de relaciones que se establecen en los centros y mejorar la calidad de las decisiones: participación en los órganos de gobierno y representación de la Universidad, 
el aprovechamiento de los servicios que tiene a su disposición y que cubren necesidades en cuanto a seguridad, fiabilidad y prestaciones, tanto individuales como colectivas (Rodríguez Gómez y González Rueda, 2000).

7. Las actividades de formación realizadas por los estudiantes en la Universidad, consideradas un elemento prioritario de las políticas de evaluación institucional en este nivel. Han de comportar los factores conceptuales, procedimentales y actitudinales necesarios para el desarrollo personal y social de los alumnos y, específicamente, para su inserción en el mercado laboral (Schmelkes, 1996 y Hernández Pina, 1997). La realización de actividades puramente académicas no cubre las necesidades formativas que, con estas características, precisan los estudiantes para desarrollarse plenamente para la vida adulta. Por esta razón hay que considerar como elementos de evaluación a la hora de constatar la calidad de una institución universitaria, qué tipo de actividades complementarias oferta la institución y qué acciones llevan a cabo los alumnos para satisfacer estas necesidades.

8. El rendimiento, medido a través del nivel alcanzado por los estudiantes (Escudero Escorza 2000: 407). Tradicionalmente, el éxito de un alumno universitario depende del nivel de rendimiento obtenido en sus estudios, entendido éste como un concepto matemático que expresa un resultado del aprendizaje promovido por el profesor y producido por el alumno, aunque Tourón (1984: 24) advierte que no todo aprendizaje es producto de la acción docente. Éste viene expresado por una calificación, cuantitativa y cualitativa, que es el reflejo de unos determinados objetivos preestablecidos o, en términos educativos, un aprendizaje.

9. La satisfacción personal, considerada como el indicador de calidad por excelencia (Apodaca y Grao 1996: 82). La satisfacción del alumno es el pilar básico de los modelos de calidad aplicados a la educación (Álvarez, 1998: 21-22). Es preciso, por lo tanto, conocer cuáles son las necesidades de los estudiantes y si estas son cubiertas por la institución. En tanto organización eminentemente compleja, esta dimensión debe atender a sus diferentes elementos como son: los sistemas de gestión, la docencia, la evaluación del rendimiento académico, los servicios, la infraestructura, el clima y la orientación para el desarrollo de la carrera para contribuir a una satisfacción a nivel general.

10. La inserción profesional de los jóvenes graduados, considerado como un elemento fundamental para su incorporación como adultos a la sociedad. (Lobato, 2001: 201). A partir de esta premisa es indudable que la transición de los estudiantes universitarios al mercado laboral ha de ser un elemento a considerar en los planes de formación y evaluación si queremos ofrecer altos niveles de calidad. Está asumida la idea de que una función de la universidad es la formación de profesionales, caracterizada por el cultivo de valores, actitudes y desarrollo de la persona en general. Por ello, hemos de considerar, en primer lugar, cuáles son los requisitos necesarios para acceder a un puesto de trabajo. Las empresas defienden actualmente un modelo de recursos humanos basado en informaciones cualitativas en torno a las características de la persona, sus capacidades y actitudes y sus conocimientos básicos para desempeñar el empleo. El éxito en la profesión vendrá dado por la conjugación de estos elementos. Desde la perspectiva del capital humano, Gil Villa (1995: 318) es consciente de que la escuela cualifica la mano de obra, en el sentido de suministrar a los alumnos las capacidades 
necesarias para desempeñar futuros puestos de trabajo. El currículo más valorado no siempre es el del candidato con mejor expediente, las empresas prefieren a quienes, a pesar de tener peores aptitudes académicas, poseen habilidades para la vida profesional como, por ejemplo, iniciativa, creatividad, flexibilidad, empatía, liderazgo y trabajo en equipo. En definitiva, la posesión de inteligencia emocional (Goleman, 1996), integrada por un conjunto de habilidades o competencias pertenecientes a la vida afectiva. A continuación, será necesario valorar el grado de conocimiento de las salidas profesionales de la titulación de los diferentes alumnos, así como advertir si la formación universitaria está dotando a los estudiantes de los recursos necesarios para enfrentarse al mundo laboral. En tercer lugar es preciso realizar una valoración de los sistemas de orientación profesional a partir de la información que los estudiantes han recibido sobre el mundo del trabajo y las salidas profesionales de las distintas titulaciones (Arbizu Bakaikoa, 1994; Echevarría, 1996 y Sanz Oro, 2001).

\section{La opinión de los estudiantes acerca de la orientación profesional como indicador de calidad en la Universidad}

Según lo expuesto hasta ahora, hemos creído importante estudiar las manifestaciones realizadas por los alumnos acerca de la consideración de la orientación profesional en la Universidad como un factor de calidad educativa.

Por ello, hemos planteado una investigación circunscrita en la Universidad de Salamanca, aplicada a una muestra representativa de 807 estudiantes matriculados en el curso académico 1999-2000.

Las variables de estudio se seleccionaron considerando aquellos elementos que condicionan a la orientación profesional como un indicador de calidad educativa. Se especificaron en:

- Salidas profesionales: grado de conocimiento de las salidas profesionales de la titulación.

- Orientación profesional: información recibida acerca de las salidas profesionales de la titulación y capacitación para acceder al mundo laboral.

- Acceso al mercado laboral: requisitos necesarios para acceder a un empleo y cualidades que se precisan para desempeñar con éxito la profesión.

Como variables de clasificación hemos tenido en cuenta el sexo de los estudiantes, la rama de especialización a la que pertenecen sus estudios y el curso académico.

La obtención de información se realizó por medio de un cuestionario compuesto por ítems de valoración escalar, validado empíricamente a través de un estudio de fiabilidad y validez (consistencia interna, estudio de discriminación y análisis factorial) que no confirmó que goza de las suficientes garantías para ser aplicado, analizado e interpretado.

\section{Resultados obtenidos: la opinión del estudiante}

Una vez aplicados los instrumentos, obtenidos los datos y realizados los análisis oportunos (estudio descriptivo e inferencial), los resultados obtenidos fueron los siguientes: 


\section{Salidas profesionales}

En este apartado únicamente hemos incluido una pregunta de valoración del grado de conocimiento sobre las distintas salidas profesionales de la titulación que están cursando. Hay que destacar cómo el $34.4 \%$ de los encuestados conocen en un grado bastante alto dichas salidas, siendo únicamente un 3.2\% quien no conoce las opciones profesionales de los estudios que está realizando. Dicha opinión no revela diferencias significativas se trate de alumnos o alumnas y de primeros y últimos cursos. Sin embargo, haciendo una estudio inferencial (análisis de varianza de un factor) en función de la rama de especialización $($ n.s. $=0.001)$, nos encontramos que el alumnado de titulaciones de Ciencias de la Salud y Técnicas muestra un grado de conocimiento de sus salidas profesionales más elevado que el resto de especialidades $(\mathrm{F}=15.696, \mathrm{p}=0.000)$.

\section{Orientación profesional}

Si acudimos a dos aspectos relacionados con la orientación profesional recibida a lo largo de los estudios (recepción de información y sentimiento de capacitación laboral), los diferentes encuestados manifestaron haber recibido poca información acerca de las profesiones que pueden ejercer tras su graduación (37.2\%). Aún así, manifiestan sentirse suficientemente capacitados para enfrentarse al mundo laboral (31.7\%) (ver Tabla 1).

TABLA 1: Orientación profesional.

\begin{tabular}{|l|c|c|c|c|c|c|c|c|}
\hline \multicolumn{1}{|c|}{$\begin{array}{c}\text { Orientación } \\
\text { profesional }\end{array}$} & $\overline{\mathbf{X}}$ & $\mathbf{S}_{\mathbf{x}}$ & $\begin{array}{c}\mathbf{1} \\
\mathbf{n a d a} \\
\mathbf{( \% )}\end{array}$ & $\begin{array}{c}\mathbf{2} \\
\mathbf{p o c o} \\
\mathbf{( \% )}\end{array}$ & $\begin{array}{c}\mathbf{3} \\
\text { suficiente } \\
(\mathbf{\%})\end{array}$ & $\begin{array}{c}\mathbf{4} \\
\text { bastante } \\
(\%)\end{array}$ & $\begin{array}{c}\mathbf{5} \\
\text { mucho } \\
(\mathbf{\%})\end{array}$ & $\mathbf{N}$ \\
\hline $\begin{array}{l}\text { Capacitado } \\
\text { mundo laboral }\end{array}$ & 2.80 & 1.07 & 11.2 & 30.1 & 31.7 & 21.4 & 5.7 & 777 \\
\hline $\begin{array}{l}\text { Recibo } \\
\text { información }\end{array}$ & 2.30 & 1.11 & 26.6 & 37.2 & 19.9 & 12.1 & 4.3 & 775 \\
\hline
\end{tabular}

No se encuentran diferencias significativas si hablamos de hombres o mujeres. Sin embargo, si diferenciamos en función del curso (ver Tabla 2), una prueba de t $($ n.s. $=0.001)$ nos confirma que son los alumnos de primero quienes evidencian que reciben poca información laboral $(\mathrm{t}=5.489, \mathrm{p}=0)$ aunque sí se sienten suficientemente capacitados para trabajar en profesiones relacionadas con su formación $(\mathrm{t}=9.948, \mathrm{p}=0.000)$. Esto puede ser debido a que son estudiantes de nuevo ingreso en fase de iniciación.

Si realizamos una distribución de estas opiniones en función de la tipología de titulaciones existentes (ver Tabla 3), apreciamos los siguientes resultados:

- El alumnado de Ciencias de la Salud y de titulaciones Técnicas ha recibido más información referente al futuro profesional que el resto de especialidades ( $\mathrm{F}=13.082$, $\mathrm{p}=0)$.

- El alumnado de carreras Técnicas se siente más capacitado para acceder al mercado laboral que los estudiantes de Humanidades $(\mathrm{F}=4.316, \mathrm{p}=0.002)$. 
TABLA 2: Orientación profesional en función del curso.

\begin{tabular}{|l|l|l|l|c|c|c|c|c|c|}
\hline $\begin{array}{c}\text { Orientación } \\
\text { profesional }\end{array}$ & Curso & $\overline{\mathbf{X}}$ & $\mathbf{S}_{\mathbf{x}}$ & $\begin{array}{c}\mathbf{1} \\
\mathbf{n a d a} \\
\mathbf{( \% )}\end{array}$ & $\begin{array}{c}\mathbf{2} \\
\mathbf{p o c o} \\
\mathbf{( \% )}\end{array}$ & $\begin{array}{c}\mathbf{3} \\
\text { suficiente } \\
\mathbf{( \% )}\end{array}$ & $\begin{array}{c}\mathbf{4} \\
\text { bastante } \\
\mathbf{( \% )}\end{array}$ & $\begin{array}{c}\mathbf{5} \\
\mathbf{m u c h o} \\
\mathbf{( \% )}\end{array}$ & $\mathbf{N}$ \\
\hline $\begin{array}{l}\text { Capacitado } \\
\text { mundo laboral }\end{array}$ & Primeros & 3.12 & 1.06 & 7 & 21 & 34.1 & 29.3 & 8.6 & 443 \\
\cline { 2 - 10 } & Últimos & 2.39 & 0.95 & 16.8 & 42.2 & 28.4 & 10.8 & 1.8 & 334 \\
\hline $\begin{array}{l}\text { Recibo } \\
\text { información }\end{array}$ & Primeros & 2.49 & 1.16 & 21.5 & 35.1 & 22.2 & 15.2 & 5.9 & 441 \\
\cline { 2 - 9 } & Últimos & 2.06 & 1.01 & 33.2 & 39.8 & 16.8 & 8.1 & 2.1 & 334 \\
\hline
\end{tabular}

TABLA 3: Orientación profesional en función de la rama de especialización.

\begin{tabular}{|l|c|c|c|c|c|c|c|c|c|c|}
\cline { 2 - 11 } \multicolumn{1}{c|}{} & \multicolumn{8}{c|}{ Rama de especialización } \\
\cline { 2 - 11 } \multicolumn{1}{c|}{} & $\begin{array}{c}\text { Ciencias de } \\
\text { la Salud }\end{array}$ & \multicolumn{2}{c|}{$\begin{array}{c}\text { Experimen- } \\
\text { tales }\end{array}$} & \multicolumn{2}{|c|}{ Humanidades } & \multicolumn{2}{c|}{$\begin{array}{c}\text { Jurídico- } \\
\text { Sociales }\end{array}$} & \multicolumn{2}{c|}{ Técnicas } \\
\hline $\begin{array}{l}\text { Orientación } \\
\text { profesional }\end{array}$ & $\overline{\mathbf{X}}$ & $\mathbf{S}_{\mathbf{x}}$ & $\overline{\mathbf{X}}$ & $\mathbf{S}_{\mathbf{x}}$ & $\overline{\mathbf{X}}$ & $\mathbf{S}_{\mathbf{x}}$ & $\overline{\mathbf{X}}$ & $\mathbf{S}_{\mathbf{x}}$ & $\overline{\mathbf{X}}$ & $\mathbf{S}_{\mathbf{x}}$ \\
\hline $\begin{array}{l}\text { Capacitado } \\
\text { mundo laboral }\end{array}$ & 2.93 & 1.18 & 2.6 & 1 & 2.55 & 1.01 & 2.79 & 1.06 & 3.09 & 1 \\
\hline $\begin{array}{l}\text { Recibo } \\
\text { información }\end{array}$ & 2.78 & 1.25 & 2.08 & 1 & 1.82 & 0.94 & 2.28 & 1.04 & 2.41 & 1.18 \\
\hline
\end{tabular}

\section{Acceso al mercado laboral}

Ofrecemos, en primer lugar, información sobre los requisitos que se plantean necesarios para acceder al mercado laboral (ver Tabla 4). De los aquí expuestos, los estudiantes (con independencia de la rama de especialización a la que pertenezcan sus estudios, entre las cuáles no se evidencian diferencias significativas) consideran muy importante la formación complementaria que hayan podido cursar a lo largo de los estudios (53.4\%), siendo también importante (40.9\%) la experiencia previa en un puesto similar, así como las cualidades personales $(37.9 \%)$.

Si realizamos una diferenciación por sexo (ver Tabla 5), nos encontramos con que las diferencias encontradas únicamente son significativas $($ n.s. $=0.05)$ y a favor de las mujeres al opinar que entre los requisitos anteriormente señalados, los más importantes para ellas son: la experiencia previa en un puesto similar $(\mathrm{t}=-1.971, \mathrm{p}=0.049)$ y la formación complementaria $(-4.186, \mathrm{p}=0.000)$.

Por otro lado, para los alumnos de primer curso es más importante la obtención del título universitario a la hora de conseguir un empleo $(\mathrm{t}=3.359, \mathrm{p}=0.001)$ y para los de último curso es significativo tener un buen aval $(\mathrm{t}=-3.478, \mathrm{p}=0.001)$ (ver Tabla 6).

En un segundo apartado, hemos considerado aquellas cualidades personales que el futuro trabajador ha de mostrar a la hora de acceder a un empleo (ver Tabla 7). Los estudiantes 
TABLA 4: Aspectos necesarios para el acceso al mercado laboral.

\begin{tabular}{|l|c|c|c|c|c|c|c|c|}
\hline Mercado laboral & $\overline{\mathbf{X}}$ & $\mathbf{\mathbf { S } _ { \mathbf { x } }}$ & $\begin{array}{c}\mathbf{1} \\
\mathbf{s i n} \\
\text { importancia } \\
(\mathbf{\%})\end{array}$ & $\begin{array}{c}\mathbf{2} \\
\mathbf{p o c o} \\
\mathbf{i m p o r t a n t e} \\
\mathbf{( \% )}\end{array}$ & $\begin{array}{c}\mathbf{3} \\
\text { importante } \\
\mathbf{( \% )}\end{array}$ & $\begin{array}{c}\mathbf{4} \\
\text { bastante } \\
\text { importante } \\
\mathbf{( \% )}\end{array}$ & $\begin{array}{c}\mathbf{5} \\
\text { muy } \\
\text { importante } \\
\mathbf{( \% )}\end{array}$ & $\mathbf{N}$ \\
\hline $\begin{array}{l}\text { 1. Título } \\
\text { universitario }\end{array}$ & 3.44 & 1.03 & 4.5 & 10.8 & 38.2 & 29.7 & 16.8 & 785 \\
\hline $\begin{array}{l}\text { 2. Experiencia } \\
\text { previa }\end{array}$ & 4.08 & 0.95 & 1.4 & 4.5 & 19.5 & 33.8 & 40.9 & 785 \\
\hline 3. Buen aval & 3.55 & 1.08 & 4.5 & 10.1 & 33.5 & 30.0 & 21.8 & 779 \\
\hline $\begin{array}{l}\text { 4. Formación } \\
\text { complementaria }\end{array}$ & 4.37 & 0.80 & 0.5 & 2.0 & 11.0 & 33.1 & 53.4 & 783 \\
\hline $\begin{array}{l}\text { 5. Cualidades } \\
\text { personales }\end{array}$ & 3.99 & 1.01 & 2.6 & 4.6 & 22.1 & 32.9 & 37.9 & 784 \\
\hline
\end{tabular}

TABLA 5: Aspectos necesarios para el acceso al mercado laboral en función del sexo.

\begin{tabular}{|c|c|c|c|c|c|c|c|c|c|}
\hline Mercado laboral & Sexo & $\overline{\mathbf{X}}$ & $\mathbf{S}_{\mathbf{x}}$ & $\begin{array}{c}1 \\
\text { nada } \\
(\%)\end{array}$ & $\begin{array}{c}2 \\
\text { poco } \\
(\%)\end{array}$ & $\begin{array}{c}3 \\
\text { suficiente } \\
(\%)\end{array}$ & $\begin{array}{c}4 \\
\text { bastante } \\
(\%)\end{array}$ & $\begin{array}{c}5 \\
\text { mucho } \\
(\%)\end{array}$ & $\mathbf{N}$ \\
\hline \multirow{2}{*}{$\begin{array}{l}\text { 1. Título } \\
\text { universitario }\end{array}$} & Hombres & 3.44 & 1.01 & 5.4 & 9.5 & 39.1 & 27.6 & 18.4 & 294 \\
\hline & Mujeres & 3.43 & 1.01 & 3.9 & 11.6 & 37.7 & 31 & 15.9 & 491 \\
\hline \multirow{2}{*}{$\begin{array}{l}\text { 2. Experiencia } \\
\text { previa }\end{array}$} & Hombres & 4 & 1 & 1.7 & 6.1 & 21.4 & 32.3 & 38.4 & 294 \\
\hline & Mujeres & 4.13 & 0.92 & 1.2 & 3.5 & 18.3 & 34.6 & 42.4 & 491 \\
\hline \multirow{2}{*}{ 3. Buen aval } & Hombres & 3.55 & 1.09 & 5.1 & 9.5 & 32 & 31.6 & 21.8 & 294 \\
\hline & Mujeres & 3.54 & 1.07 & 4.1 & 10.5 & 34.4 & 29.1 & 21.9 & 485 \\
\hline \multirow{2}{*}{$\begin{array}{l}\text { 4. Formación } \\
\text { complementaria }\end{array}$} & Hombres & 4.22 & 0.85 & 0.7 & 2.7 & 15.4 & 36.9 & 44.4 & 293 \\
\hline & Mujeres & 4.46 & 0.75 & 0.4 & 1.6 & 8.4 & 30.8 & 58.8 & 490 \\
\hline \multirow{2}{*}{$\begin{array}{l}\text { 5. Cualidades } \\
\text { personales }\end{array}$} & Hombres & 3.93 & 1.04 & 3.1 & 5.1 & 24.2 & 30.7 & 36.9 & 293 \\
\hline & Mujeres & 4.02 & 0.98 & 2.2 & 4.3 & 20.8 & 34.2 & 38.5 & 491 \\
\hline
\end{tabular}

han valorado todas muy positivamente pero, de entre las diez opciones planteadas, las más importante ha sido la facilidad para la comunicación (48.6\%), seguida por la capacidad para tomar decisiones (46.9\%) y la iniciativa (44.3\%).

Tomando como variable de clasificación el sexo de los alumnos, hemos encontrado diferencias significativas a favor de las mujeres en todas las cualidades aquí indicadas a excepción de tres: dinamismo, trabajo en equipo y liderazgo, donde son ambos sexos quienes manifiestan niveles similares de relevancia. 
TABLA 6: Aspectos necesarios para el acceso al mercado laboral en función del curso.

\begin{tabular}{|c|c|c|c|c|c|c|c|c|c|}
\hline Mercado laboral & Curso & $\overline{\mathbf{x}}$ & $\mathbf{S}_{\mathbf{x}}$ & $\begin{array}{c}1 \\
\text { nada } \\
(\%)\end{array}$ & $\begin{array}{c}2 \\
\text { poco } \\
(\%)\end{array}$ & $\begin{array}{c}3 \\
\text { suficiente } \\
(\%)\end{array}$ & $\begin{array}{c}4 \\
\text { bastante } \\
(\%)\end{array}$ & $\begin{array}{c}5 \\
\text { mucho } \\
(\%)\end{array}$ & $\mathbf{N}$ \\
\hline \multirow{2}{*}{$\begin{array}{l}\text { 1. Título } \\
\text { universitario }\end{array}$} & Primeros & 3.54 & 1.02 & 3.3 & 10.3 & 34.4 & 32.8 & 19.2 & 448 \\
\hline & Últimos & 3.29 & 1.03 & 5.9 & 11.6 & 43.3 & 25.5 & 13.6 & 337 \\
\hline \multirow{2}{*}{$\begin{array}{l}\text { 2. Experiencia } \\
\text { previa }\end{array}$} & Primeros & 4.04 & 0.98 & 1.1 & 6.5 & 20.3 & 31.7 & 40.4 & 448 \\
\hline & Últimos & 4.14 & 0.9 & 1.8 & 1.8 & 18.4 & 36.5 & 41.5 & 337 \\
\hline \multirow{2}{*}{ 3. Buen aval } & Primeros & 3.43 & 1.07 & 4.9 & 12.1 & 36.3 & 28.3 & 18.4 & 446 \\
\hline & Últimos & 3.7 & 1.06 & 3.9 & 7.5 & 29.7 & 32.4 & 26.4 & 333 \\
\hline \multirow{2}{*}{$\begin{array}{l}\text { 4. Formación } \\
\text { complementaria }\end{array}$} & Primeros & 4.35 & 0.82 & 0.2 & 2.9 & 11.9 & 31.8 & 53.2 & 447 \\
\hline & Últimos & 4.39 & 0.77 & 0.9 & 0.9 & 9.8 & 34.8 & 53.6 & 336 \\
\hline \multirow{2}{*}{$\begin{array}{l}\text { 5. Cualidades } \\
\text { personales }\end{array}$} & Primeros & 3.99 & 1.03 & 2.7 & 4.7 & 23.4 & 29.7 & 39.5 & 448 \\
\hline & Últimos & 3.99 & 0.98 & 2.4 & 4.5 & 20.2 & 37.2 & 35.7 & 336 \\
\hline
\end{tabular}

TABLA 7: Cualidades para acceder a un empleo.

\begin{tabular}{|c|c|c|c|c|c|c|c|c|}
\hline Cualidades & $\overline{\mathbf{X}}$ & $\mathbf{S}_{\mathbf{x}}$ & $\begin{array}{c}1 \\
\sin \\
\text { importancia } \\
(\%)\end{array}$ & $\begin{array}{c}2 \\
\text { poco } \\
\text { importante } \\
(\%)\end{array}$ & $\begin{array}{c}3 \\
\text { importante } \\
(\%)\end{array}$ & $\begin{array}{c}4 \\
\text { bastante } \\
\text { importante } \\
(\%)\end{array}$ & $\begin{array}{c}5 \\
\text { muy } \\
\text { importante } \\
(\%)\end{array}$ & $\mathbf{N}$ \\
\hline 1. Dinamismo & 3.71 & 0.92 & 1.0 & 7.4 & 32.0 & 38.4 & 21.1 & 768 \\
\hline $\begin{array}{l}\text { 2. Trabajo en } \\
\text { equipo }\end{array}$ & 3.90 & 0.93 & 1.0 & 5.7 & 24.9 & 38.6 & 29.7 & 770 \\
\hline $\begin{array}{l}\text { 3. Capacidad } \\
\text { tomar decisiones }\end{array}$ & 4.23 & 0.89 & 1.2 & 3.4 & 13.8 & 34.8 & 46.9 & 768 \\
\hline $\begin{array}{l}\text { 4. Facilidad } \\
\text { comunicación }\end{array}$ & 4.31 & 0.79 & 0.6 & 0.9 & 13.4 & 36.5 & 48.6 & 770 \\
\hline $\begin{array}{l}\text { 5. Capacidad de } \\
\text { aprendizaje }\end{array}$ & 4.15 & 0.87 & 1.2 & 3.2 & 15.1 & 40.5 & 40.0 & 770 \\
\hline 6. Iniciativa & 4.16 & 0.92 & 1.2 & 3.9 & 16.5 & 34.2 & 44.3 & 770 \\
\hline 7. Creatividad & 4.03 & 0.97 & 1.4 & 5.3 & 20.7 & 34.2 & 38.4 & 769 \\
\hline 8. Tacto relaciones & 4.10 & 0.91 & 0.9 & 4.3 & 19.0 & 35.8 & 40.1 & 769 \\
\hline $\begin{array}{l}\text { 9. Planificación/ } \\
\text { organización }\end{array}$ & 4.18 & 0.85 & 0.7 & 3.5 & 14.2 & 40.6 & 41.1 & 769 \\
\hline 10. Liderazgo & 3.45 & 1.12 & 5.1 & 14.7 & 31.3 & 28.3 & 20.5 & 769 \\
\hline
\end{tabular}


No ocurre lo mismo con el curso, ya que prácticamente todos los estudiantes manifiestan la misma importancia para todas las cualidades (n.s. $=0.01)$, a excepción del trabajo en equipo $(\mathrm{t}=-2.612, \mathrm{p}=0.009)$ y el liderazgo $(\mathrm{t}=-4.32, \mathrm{p}=0.000)$, que son mejor valorados por los alumnos de últimos años de carrera.

Sin embargo, diferenciando en función de la rama de especialización (ver Tabla 8), hemos encontrado datos como los siguientes:

- Los alumnos de carreras Técnicas consideran como cualidad más importante el trabajo en equipo $(\mathrm{F}=6.841, \mathrm{p}=0)$, y la capacidad para tomar decisiones $(\mathrm{F}=3.956$, $\mathrm{p}=0.003)$, cualidades compartidas con las titulaciones Jurídico-Sociales.

- Por otro lado, los estudiantes de titulaciones Técnicas también consideran imprescindible la capacidad de planificación y organización $(\mathrm{F}=4.517, \mathrm{p}=0.001)$.

- Por último, hemos de señalar que los estudiantes de carreras Jurídico-Sociales también creen que es importante para llegar a tener éxito en una profesión el tacto en la relaciones personales $(\mathrm{F}=5.834, \mathrm{p}=0)$.

TABLA 8: Cualidades para acceder a un empleo en función de la rama de especialización.

\begin{tabular}{|c|c|c|c|c|c|c|c|c|c|c|}
\hline \multirow[b]{3}{*}{ Cualidades } & \multicolumn{10}{|c|}{ Rama de especialización } \\
\hline & \multicolumn{2}{|c|}{$\begin{array}{l}\text { Ciencias de } \\
\text { la Salud }\end{array}$} & \multicolumn{2}{|c|}{$\begin{array}{l}\text { Experimen- } \\
\text { tales }\end{array}$} & \multicolumn{2}{|c|}{$\begin{array}{l}\text { Humani- } \\
\text { dades }\end{array}$} & \multicolumn{2}{|c|}{$\begin{array}{l}\text { Jurídico- } \\
\text { Sociales }\end{array}$} & \multicolumn{2}{|c|}{ Técnicas } \\
\hline & $\overline{\mathbf{X}}$ & $\mathbf{S}_{\mathbf{x}}$ & $\overline{\mathbf{x}}$ & Sx & $\overline{\mathbf{X}}$ & $\mathbf{S}_{\mathbf{x}}$ & $\overline{\mathbf{X}}$ & $\mathbf{S}_{\mathbf{x}}$ & $\overline{\mathbf{X}}$ & $\mathbf{S}_{\mathbf{x}}$ \\
\hline 1. Dinamismo & 3.84 & 0.94 & 3.57 & 0.82 & 3.62 & 0.99 & 3.68 & 0.9 & 3.87 & 0.92 \\
\hline $\begin{array}{l}\text { 2. Trabajo en } \\
\text { equipo }\end{array}$ & 3.91 & 0.9 & 3.68 & 0.87 & 3.61 & 0.98 & 3.95 & 0.91 & 4.23 & 0.89 \\
\hline $\begin{array}{l}\text { 3. Capacidad tomar } \\
\text { decisiones }\end{array}$ & 4.23 & 0.91 & 4.11 & 0.88 & 3.96 & 1.01 & 4.28 & 0.85 & 4.4 & 0.81 \\
\hline $\begin{array}{l}\text { 4. Facilidad } \\
\text { comunicación }\end{array}$ & 4.29 & 0.93 & 4.19 & 0.72 & 4.31 & 0.8 & 4.38 & 0.71 & 4.21 & 0.83 \\
\hline $\begin{array}{l}\text { 5. Capacidad de } \\
\text { aprendizaje }\end{array}$ & 4.12 & 0.93 & 4.15 & 0.91 & 4.1 & 0.96 & 4.13 & 0.87 & 4.34 & 0.65 \\
\hline 6. Iniciativa & 4.21 & 0.89 & 3.93 & 0.98 & 3.91 & 1.12 & 4.23 & 0.85 & 4.3 & 0.85 \\
\hline 7. Creatividad & 3.98 & 1.04 & 3.81 & 1.06 & 3.86 & 1.07 & 4.11 & 0.88 & 4.15 & 0.94 \\
\hline 8. Tacto relaciones & 4.24 & 0.94 & 3.86 & 0.98 & 3.92 & 0.98 & 4.2 & 0.84 & 3.87 & 0.93 \\
\hline $\begin{array}{l}\text { 9. Planificación/ } \\
\text { organización }\end{array}$ & 4.18 & 0.86 & 4 & 0.82 & 3.97 & 1.05 & 4.21 & 0.8 & 4.42 & 0.7 \\
\hline 10. Liderazgo & 3.36 & 1.13 & 3.19 & 1.13 & 3.21 & 1.17 & 3.56 & 1.07 & 3.59 & 1.17 \\
\hline
\end{tabular}

Un 5.6\% de estudiantes del total de la muestra consideran necesarias otra serie de cualidades para acceder al mercado laboral, complementarias a las reseñadas en el apartado an- 
terior. Estas se centran básicamente en cualidades como la constancia, la responsabilidad, el esfuerzo, el respeto y la honestidad, en la posesión de cocimientos básicos de la profesión y específicos como informática e idiomas. En menor medida, consideran preciso disponer de un buen expediente académico y una buena presencia.

\section{Conclusiones}

Como ya apuntaron Arbizu Bakaikoa (1994) en la Universidad del País Vasco y Sánchez García (1999) en la UNED, el alumnado demandaba de un modo urgente una serie de acciones orientadoras centradas fundamentalmente en aspectos académicos y profesionales, pudiendo comprobar esta necesidad en la Universidad de Salamanca. Los datos obtenidos en nuestra investigación ponen de manifiesto la escasa información recibida por los estudiantes sobre las salidas profesionales de su titulación, aunque manifiesten sentirse suficientemente capacitados para enfrentarse al mundo laboral.

Igualmente, hemos podido constatar que la opinión de los alumnos es que, para conseguir un empleo, es indispensable disponer de conocimientos complementarios (idiomas e informática principalmente). También son muy importantes la experiencia previa en un puesto similar y las cualidades personales. Villar et al (2001) unen a estos requisitos la confección de un sistema de contactos para obtener el primer puesto de trabajo.

De las cualidades valoradas por los alumnos e indispensables para acceder al mercado laboral, destacan la facilidad para la comunicación, la capacidad para tomar decisiones y la iniciativa. Castillo Cevallos (2000) advierte la necesidad de una formación en valores, la capacidad de actuar con autonomía, la adaptación y la preocupación por la propia formación permanente, como requisitos básicos del futuro trabajador. Siendo conscientes de estas prioridades, las mujeres consideran que también son necesarias cualidades como el dinamismo, el trabajo en equipo y el liderazgo, compartidas por los estudiantes de último año de carrera. En lo que respecta a las especialidades, los alumnos de carreras Técnicas consideran que son precisas cualidades como el trabajo en equipo, la capacidad para tomar decisiones, la planificación y la organización. Por su parte también destacan las opiniones de los alumnos de la especialidad Jurídico-Social, que señalan al trabajo en equipo, la toma de decisiones y el tacto en las relaciones personales como cualidades complementarias a las ya citadas para tener éxito en la profesión.

A la vista de estos comentarios, podemos afirmar que los alumnos encuestados, a parte de plantear la demanda de orientación como un elemento que favorece su integración sociolaboral, manifiestan la necesidad de disponer de servicios universitarios destinados a cubrir este objetivo. Es por ellos razones, que este grupo de estudiantes, consideran a la orientación profesional en la universidad como un indicador esencial para valorar la calidad de esta institución, considera a la orientación profesional en la Universidad como un indicador esencial para valorar la calidad de esta institución.

\section{Referencias bibliográficas}

Abrile, M. (1994). "Nuevas demandas a la educación, la institución escolar y la profesionalización de los docentes". Revista Iberoamericana de Educación, 5, pp. 11-43. 
Álvarez Rojo, V. et al. (1999). "El rendimiento académico en la universidad desde la perspectiva del alumnado". Revista Española de Orientación y Psicopedagogía, 10 (17), pp. 23-42.

Álvarez, M. (1998). El liderazgo de la calidad total. Madrid: Escuela Española.

Apodaca, P. M. y Grao, J. (1996). “Autoevaluación, planificación estratégica y calidad total”. En F. J. Tejedor y J. L. Rodríguez Diéguez (Eds.). Evaluación educativa II: Evaluación Institucional. Fundamentos teóricos y aplicaciones prácticas. Salamanca: Instituto Universitario de Ciencias de la Educación de la Universidad de Salamanca.

Apodaca, P. y Lobato, C. (Eds.) (1997). Calidad en la universidad: orientación y evaluación. Barcelona: Laertes.

Arbizu Bakaikoa, F. (1994). "La labor orientadora del profesor universitario desde la perspectiva del alumnado y del profesorado". Revista de Investigación Educativa, 23, pp. 614-622.

Astin, A. W. y Henson, J. W. (1977). "New meassures of college selectivity". Research in Higher Edcation, 6, pp. 1-8.

Atkins, J. M.; Beattie, J. y Dockrell, W. B. (1993). Assessment Issues in Higher Education. Sheffield, Inglaterra: Emploiment Department.

Ball, R. y Halwachi, J. (1987). "Performance indicators in higher education". Higher Education, 16, pp. 393-405.

Bricall, J. M. (2000). Informe Universidad 2000. Madrid: Conferencia de Rectores de las Universidades Españolas.

Castillo Cevallos, G. (2000). De la Universidad al puesto de trabajo. Estrategias y recursos para acceder al primer empleo. Madrid: Pirámide.

Chadwick, C. B. y Thorne de Trelles, C. (1998). 'Calidad de la Educación. Ponderación de variables.

International Society for Performance Improvement. http://www.pignc-ispi.com/articles/quality/ chadwick-educacion.htm\#op (18 de enero de 2002).

Díaz Allué, M. T. (1989). La orientación universitaria ante la problemática académica y profesional del estudiante. Madrid: Narcea.

Echevarría, B. (1996). Presentación de las Actas de las Jornadas sobre Orientación Académica y Profesional en la Universidad. Barcelona: Universitat de Barcelona.

Escudero Escorza, T. (2000). "La evaluación y mejora de la enseñanza en la universidad: otra perspectiva". Revista de Investigación Educativa, 18 (2), pp. 405-416.

García Basallote, M. et al. (1999). "Un proyecto para la mejora de la orientación universitaria: el programa Tutor". Revista de Investigación Educativa, 17 (2), pp. 401-406.

Gento Palacios, S. (1996). Instituciones educativas para la Calidad Total. Madrid: La Muralla.

Gil Villa, F. (1995). "El estudiante como actor racional. Objeciones a la teoría del capital humano". Revista de Educación, 306, pp. 315-327.

Goleman, D. (1996). Inteligencia emocional. Barcelona: Kairós.

Haveman, R. F. y Wolfe, B. L. (1984). "Schooling and economic well-being: the role of nonmarket effects". Journal of human Resources, 19 (3), pp. 377-407. 
Hernández Pina, F. (1997). "La evaluación de los alumnos en el contexto de la calidad de las universidades". Revista de Investigación Educativa, 14 (2), pp. 25-50.

López Franco, E. y Oliveros, L. (1999). "La acción tutorial de la función docente universitaria”. Revista Española de Orientación y Psicopedagogía, 10 (17), pp. 83-98.

López Mojarro, M. (1999). A la calidad por la evaluación. La evaluación de centros docentes. Madrid: Escuela Española.

Maslow, A. (1991). Motivación y personalidad. Madrid: Díaz de Santos.

Moncada, A. (1971). Administración universitaria. Introducción sistemática a la enseñanza superior. Madrid: ICE Universidad Complutense de Madrid.

Mora Ruiz, J. G. (1991). Calidad y rendimiento en las instituciones universitarias. Madrid: Consejo de Universidades.

OCDE (1991). Escuelas y calidad de la enseñanza. Informe internacional. Barcelona: Paidós / Ministerio de Educación y Ciencia.

Real Decreto 1947/1995, de 1 de diciembre, por el que se establece el Plan Nacional de Evaluación de la Calidad de las Universidades (Boletín Oficial del Estado número 294, de 9 de diciembre de 1995).

Rodríguez Diéguez, A. (2002). "La orientación en universidad: ámbitos de intervención, metas y objetivos, roles y funciones del orientador. Análisis de una estrategia de integración de la orientación en el currículum universitario". En V. Álvarez Rojo y A. Lázaro Martínez (Coords). Calidad de las Universidades y Orientación Universitaria (pp. 171-194). Málaga: Aljibe.

Rodríguez Espinar, S. (1989). "Problemática y tendencias de la orientación universitaria". La reforma educativa: un reto para la orientación. V Jornadas Nacionales de Orientación Educativa, Valencia.

Rodríguez Gómez, G. y González Rueda, A.G. (Coords.) (2000). Calidad en los Servicios Universitarios. Cádiz: Universidad de Cádiz.

Rodríguez, G. (2002). "El reto de enseñar hoy en la Universidad”. En V. Álvarez Rojo y A. Lázaro Martínez (Coords). Calidad de las Universidades y Orientación Universitaria. (pp. 9-13) Málaga: Aljibe.

Romero, S. y Sobrado, L. (2002). "Orientación para la carrera profesional". En V. Álvarez Rojo y A. Lázaro Martínez (Coords). Calidad de las Universidades y Orientación Universitaria. (pp. 305-324) Málaga: Aljibe.

San Fabián Maroto, J. L. (1997). La experiencia participativa de los estudiantes en la Universidad. Madrid: Centro de Investigación y Documentación Educativa.

Sánchez García, M. F. (1999). Necesidades y servicios de orientación universitaria en la Comunidad de Madrid. Madrid: UNED. mide.

Sanz Oro, R. (2001). Orientación psicopedagógica y calidad educativa. Madrid: Pirá-

Schmelkes, S. (1996). "La evaluación de los centros escolares". Programa de Evaluación de la Calidad de la Educación, Documento 3. http://www.ince.mec.es/cumbre/d1-03.htm (15 de julio de 2001). 
Touron Figueroa, J. (1984). Factores del rendimiento académico en la Universidad. Pamplona: Universidad de Navarra.

Villar, E. et al. (2001). "Educación universitaria y construcción de redes sociales como estrategia de inserción profesional". Revista de Investigación Educativa, 19 (1), pp. 221-247.

Fecha de recepción: 25-03-2003

Fecha de revisión: 06-07-2004

Fecha de aceptación: 05-11-2004 